\title{
Editorial
}

\section{Wide-Bandgap Semiconductors: Nanostructures, Defects, and Applications}

\author{
Meiyong Liao, ${ }^{1}$ Thomas Stergiopoulos, ${ }^{2}$ Jose Alvarez, \\ Surojit Chattopadhyay, ${ }^{4}$ and Guihua Zhang ${ }^{5}$ \\ ${ }^{1}$ Optical and Electronic Materials Unit, National Institute for Materials Science, Namiki 1-1, Tsukuba, Ibaraki 3050044, Japan \\ ${ }^{2}$ Department of Physics, University of Oxford, Clarendon Laboratory, Parks Road, Oxford OX13PU, UK \\ ${ }^{3}$ Laboratoire de Genie Electrique de Paris (LGEP), CNRS UMR 8507, Supelec, Université Paris-Sud, Université Paris 6, \\ 11 rue Joliot Curie, Plateau de Moulon, 91192 Gif-sur-Yvette, France \\ ${ }^{4}$ Institute of Biophotonics, National Yang-Ming University, No. 155, Section 2, Li-Nong Street, Taipei 112, Taiwan \\ ${ }^{5}$ Research Institute of Science for Safety and Sustainability, National Institute of Advanced Industrial Science and Technology (AIST), \\ Onogawa 16-1, Tsukuba, Ibaraki 3058569, Japan
}

Correspondence should be addressed to Meiyong Liao; meiyong.liao@nims.go.jp

Received 6 August 2015; Accepted 6 August 2015

Copyright (C) 2015 Meiyong Liao et al. This is an open access article distributed under the Creative Commons Attribution License, which permits unrestricted use, distribution, and reproduction in any medium, provided the original work is properly cited.

Nanostructured wide-bandgap semiconductors (NWS), such as III-nitrides, $\mathrm{SiC}, \mathrm{ZnO}, \mathrm{TiO}_{2}$, diamond, $\mathrm{AlN}$, and $\mathrm{BN}$, have attracted intensive research attention owing to prospective applications in solid-state lighting, solar cells, power electronics, sensors, spintronics, and MEMS/NEMS. These nanostructured semiconductors exhibit tremendous advantages in terms of power capability, energy conversion efficiency, optical properties, radiation strength, high temperature, and frequency operation. Although great progress has been achieved in the synthesis of the NWS materials and promising device applications have been demonstrated since the new century, much further research in the crystallinity improvement, electronic structure control, impurities doping, and devices design need to be carried out. The growth dynamics and the defect physics of NWS should be better understood to push forward their potential applications.

This special issue is focused on recent research progress in wide-bandgap semiconductors materials including novel growth strategies of NWS materials, the electronic structure tailoring for functionalization, novel devices concepts, devices physics, and applications in various fields.

By using first-principles calculations based on the density functional theory, D. Ma et al. investigated the defects in gallium arsenide. A deep donor level of $0.85 \mathrm{eV}$ below the conduction band minimum on the gallium arsenide crystal surface was disclosed, while the lowest donor level of the defect inside the gallium arsenide bulk was $0.83 \mathrm{eV}$. The calculations also predicted that the formation energies of internal and surface defects were around $2.36 \mathrm{eV}$ and $5.54 \mathrm{eV}$, respectively. They concluded that the formation of defect within the crystal was easier than that on surface. This work would assist in tailoring the electronic structures of gallium arsenide, thus favouring the development of highperformance electronic devices.

Two papers on $\mathrm{ZnO}$ are contributed to this issue. One is on thin film $\mathrm{ZnO}$ and the other on 1-dimensional $\mathrm{ZnO}$ nanorods. L. Meng et al. report 2-dimensional electrongas (2DEG) properties of a $\mathrm{Zn}$ polar $\mathrm{ZnMgO} / \mathrm{MgO} / \mathrm{ZnO}$ structure with low $\mathrm{Mg}$ composition layer $(x=0.05)$ grown on a-plane (11-20) sapphire by radical-source laser molecular beam epitaxy. They observed that the insertion of a thin $(1 \mathrm{~nm}) \mathrm{MgO}$ layer between $\mathrm{ZnMgO}$ and $\mathrm{ZnO}$ layers in the $\mathrm{ZnMgO} / \mathrm{ZnO}$ 2DEG structures resulted in an increase of the sheet density and affected the electron mobility slightly. The resultant carrier concentration was as high as $1.1 \times$ $10^{13} \mathrm{~cm}^{-2}$ and the Hall mobility was as high as $3090 \mathrm{~cm}^{2} / \mathrm{Vs}$ at $10 \mathrm{~K}$ and $332 \mathrm{~cm}^{2} / \mathrm{Vs}$ at RT. The authors also calculated the dependence of carrier sheet density of the $2 \mathrm{DEG}$ on $\mathrm{ZnMgO}$ 
layer thickness, which was consistent with the experiments. $\mathrm{Y}$. Zhang et al. report the high-quality nitrogen-doped $\mathrm{ZnO}$ nanorods selectively grown on patterned and bare $\mathrm{ZnO}$ templates by the combination of nanoimprint lithography and chemical vapor transport methods. It is encouraging that the grown nanorods were uniform in size and orientation with controllable density and surface-to-volume ratio. They also analysed the structural and optical properties of the nitrogen-doped $\mathrm{ZnO}$ nanorods in detail.

Both fundamental and practical researches on III-nitride are presented. The mechanism of dislocations and interface roughness effect on the 2DEG mobility limitation in an AlGaN/AlN/GaN heterostructure is reported theoretically and experimentally by L. Meng et al. They observed that the charge impurities scattering limited the 2DEG mobility at low and moderate temperatures, followed by the dislocation and interface roughness scattering, whereas the polar optical phonon scattering was dominant above $200 \mathrm{~K}$. S.-L. Jeng et al. established a standardized electrical measurement procedure for GaN power transistors, which provided necessary information for designers. In addition, the authors proposed an isolated gate driver detection method for sorting the uniformity of the power transistors from the option of the turn-off characteristic. They provided an equivalent-circuit model for GaN FETs on the basis of the measured electrical characteristics. In order to inhibit the effect of current crowding and to improve the yield of high-voltage single-chip GaN light-emitting diode (LED), W. Wang et al. put forward a parallel and series network structure. Based on this design, a 3W-LED chip of 24 parallel stages was demonstrated at the current of $500 \mathrm{~mA}$ with a $\mathrm{mm}^{2}$ large area.

The recent work on semiconductor diamond is also shown. W. Wang et al. report a Zr-gate diamond field-effect transistor with $\mathrm{SiN}_{x}$ dielectric layers (SD-FET). The FET utilized the p-type surface conductivity of intrinsic diamond terminated by surface hydrogen. The fabricated FET was normally on with a sheet hole density of $2.17 \times 10^{13} \mathrm{~cm}^{-2}$ and mobility of $24.4 \mathrm{~cm}^{2} \cdot \mathrm{V}^{-1} \cdot \mathrm{s}^{-1}$. The drain current density was less than $10 \mathrm{~mA} / \mathrm{mm}$. H. Li et al. present a review of the recent progress in three diamond nanostructures, 2-dimensional diamond film, nanostructural surface on diamond, and diamond nanoheterojunction. The future challenges in these topics were addressed.

In addition, controlled growth of Scheelite-type $\mathrm{BaMoO}_{4}$ microstructures was reported by Y. Yin et al. And K. M. Lee et al. investigated the mechanism and kinetics for photocatalytic oxidation degradation by using $\mathrm{ZnO}$ as a photocatalyst.

Despite limited submissions, this special issue covers a wide range of topics from fundamental theoretical calculation, materials growth, and device physics to applications on wide-bandgap semiconductors nanostructures. The importance of wide-bandgap semiconductor is further demonstrated by the 2014 Nobel Laureate in Physics to Professor Isamu Akasaki, Professor Hiroshi Amano, and Professor Shuji Nakamura for their contributions in LEDs based on IIInitrides. We hope that this special issue, launched in August 2014, is timely to the readers for references in the related research fields.

\section{Acknowledgments}

The editors would like to sincerely express their thanks to the authors for the submissions to this special issue. We also greatly appreciate the reviewers for their constructive comments on the manuscripts.
Meiyong Liao

Thomas Stergiopoulos Jose Alvarez

Surojit Chattopadhyay Guihua Zhang 

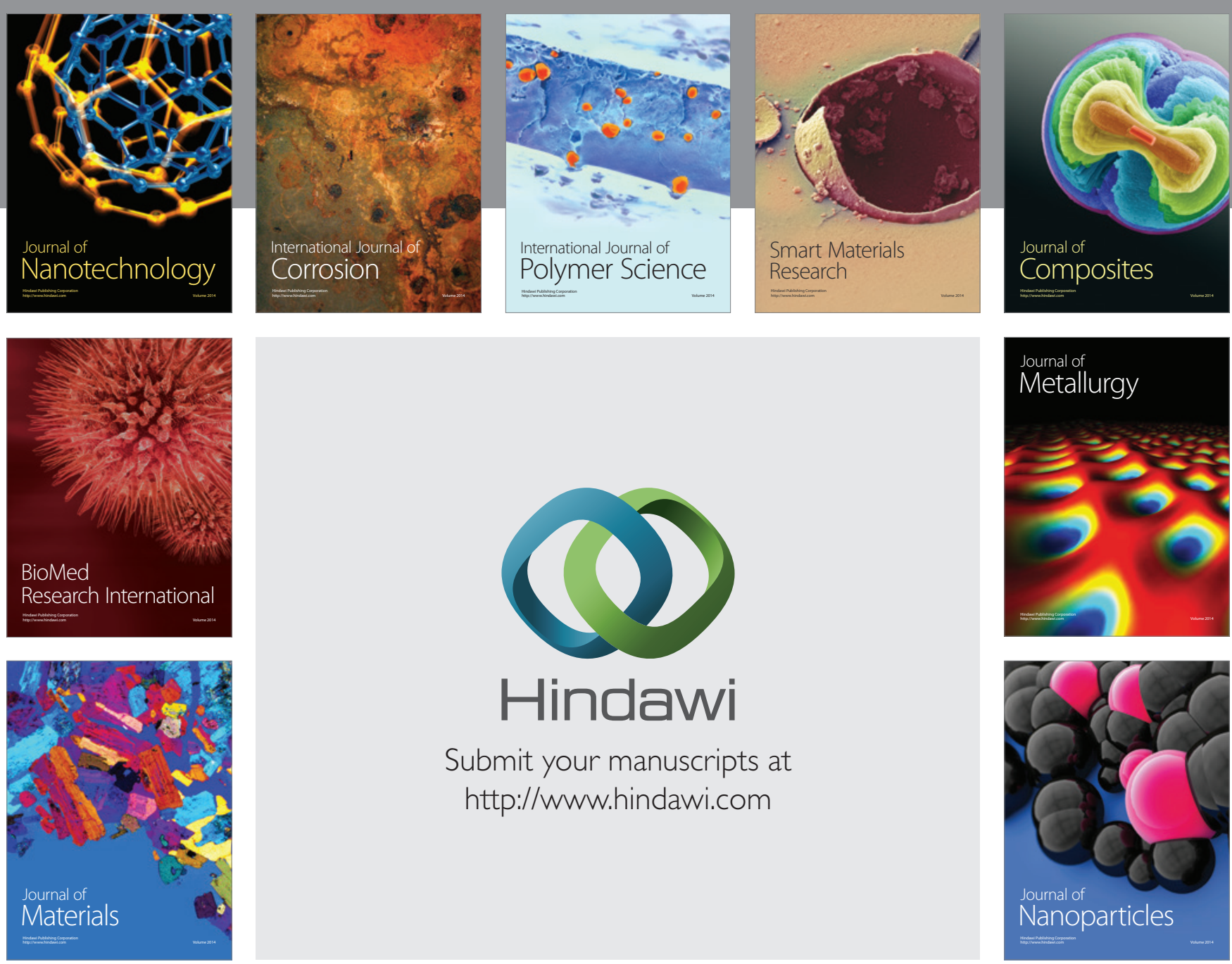

Submit your manuscripts at http://www.hindawi.com
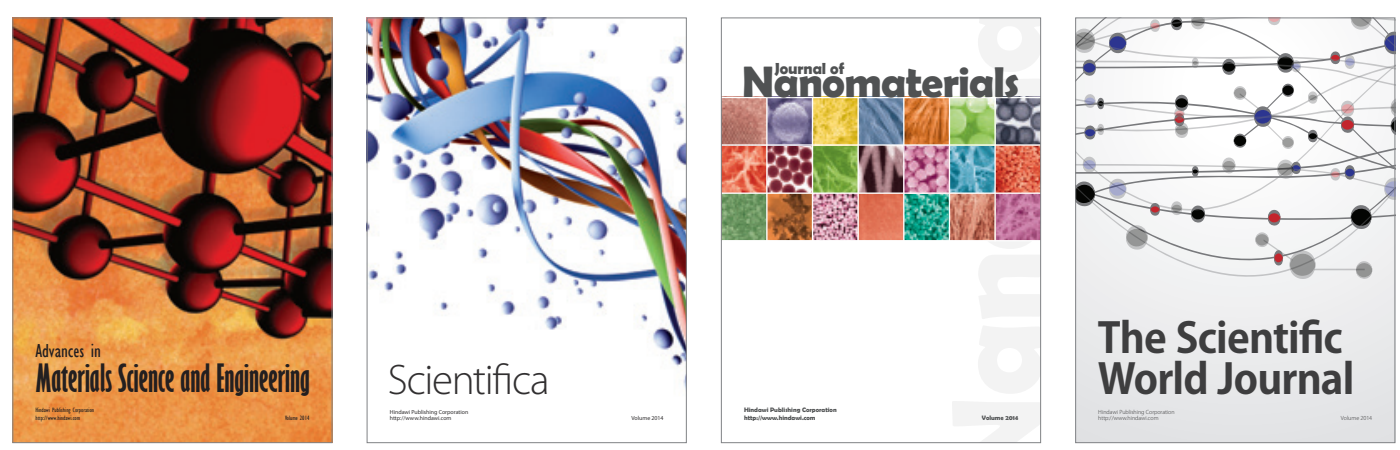

\section{The Scientific World Journal}
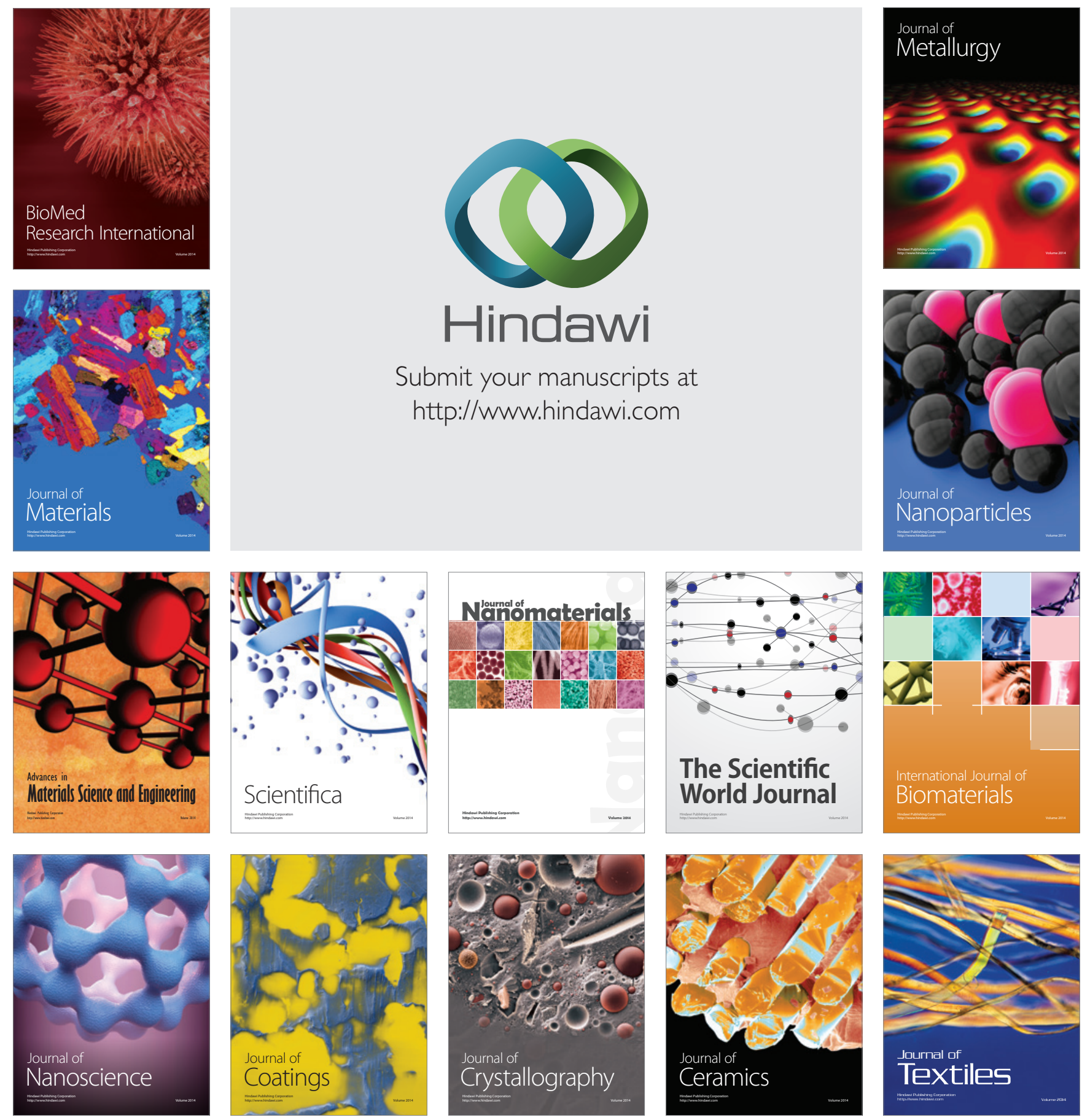